\title{
Differential Effect of Nobiletin and Tangeretin on the Antioxidant Activity and Levels of $\alpha$-Tocopherol and Retinol in Rats
}

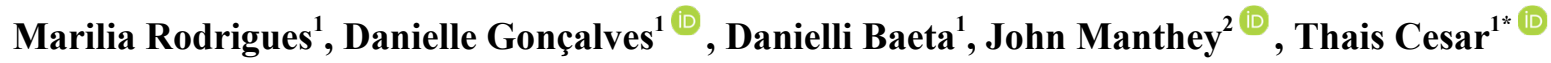 \\ ${ }^{1}$ Pharmacy School, Sao Paulo State University-UNESP Araraquara, SP 14802-901, Brazil \\ ${ }^{2}$ United States Horticultural Research Laboratory, ARS, USDA Fort Pierce, FL 34945, United States \\ E-mail: thais.cesar@unesp.br
}

Received: 13 July 2021; Revised: 30 September 2021; Accepted: 08 October 2021

\begin{abstract}
Polymethoxylated flavones (PMFs) have been associated with increased antioxidant activity in animal models. The effects of tangeretin (TAN) and nobiletin (NOB) on antioxidant activity in the blood and liver of rats were evaluated. Groups of rats were treated with $200 \mathrm{mg} / \mathrm{kg}$ bw/day of TAN or NOB, or placebo, for 15 days. Parental compounds and their metabolites were assessed in the liver by chromatographic analysis, in addition to $\alpha$-tocopherol and retinol in the blood serum. Both TAN and NOB supplements were able to reduce malonaldehyde (MDA) in the rat's blood by $22 \%$ and $18 \%$, respectively, but only NOB increased redox reaction by $3 \%$. Blood levels of retinol and $\alpha$-tocopherol increased under TAN by $59 \%$ and $20 \%$, respectively, but were not affected by NOB. Eight NOB metabolites were detected in the liver, but only two TAN metabolites were identified in low concentration. In conclusion, NOB improved antioxidant capacity and reduced lipid peroxidation, while increased levels of retinol and $\alpha$-tocopherol after TAN supplement may have contributed to decreased blood lipid peroxidation.
\end{abstract}

Keywords: nobiletin, tangeretin, metabolites, antioxidant activity, retinol, alpha-tocopherol

\section{Introduction}

Blood levels of antioxidants can vary in response to normal oxidative metabolism, controlling the production of reactive oxygen species (ROS) that damage cell molecules. However, in the course of the infection or inflammation that follows most human diseases, there is a greater demand for antioxidants to neutralize oxidative damage [1]. Diets containing plenty of fruits and vegetables may meet those demands, but dietary supplements can be designed to offer a variety of antioxidants in ample amounts. Antioxidants extracted from citrus fruits, such as vitamin C, carotenoids, flavanones (hesperidin and naringenin), and polymethoxyflavones (PMFs), such as nobiletin (NOB) and tangeretin (TAN), can be used as nutraceuticals to benefit health [2].

Currently, the use of supplements by the general population has been encouraged by broad segments of society, such as health professionals, the pharmaceutical industry and social networks, as an alternative and faster way to improve well-being by combating conditions associated with unhealthy and stressful lifestyles. Despite apparent benefits, the extensive use of supplemental antioxidants can unbalance the redox state, causing negative effects, depending on the dose and the physiological state [1]. The benefits of NOB and TAN supplementation are associated

Copyright (C2021 Thais Cesar, et al.

DOI: https://doi.org/10.37256/fse.2220211038

This is an open-access article distributed under a CC BY license

(Creative Commons Attribution 4.0 International License)

https://creativecommons.org/licenses/by/4.0/ 
with their ability to block oxidative stress and modulate enzymes that cause the accumulation of free radicals and ROS [3-5]. This leads to inhibition of early stages of inflammation and overproduction of pro-inflammatory cytokines such as tumor necrosis factor alpha (TNF- $\alpha$ ), interleukin $1 \mathrm{~b}$ (IL-1b), interleukin 6 (IL-6), and nitric oxides (NO) [6-7]. These combined effects improve overall redox balance and decrease systemic and local inflammation [8], mitigating damage caused by disease-or drug-induced oxidative stress [9].

Although the protective activity of PMFs has been demonstrated in animals [3, 9-10] and humans [11], the effect of regular ingestion of higher doses under physiological conditions is still unknown. It was suggested that the indiscriminate use of antioxidants, not clinically prescribed, could disturb the oxidative balance, limit the extent of its effect, or even act as a pro-oxidant agent [12]. Therefore, it is relevant to know whether supplementation of a given dose of NOB and TAN, under physiological conditions, will contribute to the stability of the antioxidant system or lead to undesirable effects [13]. More research is warranted for recommendation of these supplements, aimed at maintaining health, preventing diseases, or treating a specific pathology [11].

The main objective of this study was to evaluate biological parameters related to the antioxidant activity of NOB and TAN, after systematic supplementation of $200 \mathrm{mg} / \mathrm{kg}$ of body weight (bw)/day for 15 days in apparently healthy animals. This translated dose for an average $70 \mathrm{~kg}$ person is about $2.3 \mathrm{~g} /$ day [14], and it is considered a high dose since the general recommendation of citrus bioflavonoids for humans is around $500 \mathrm{mg} / \mathrm{d}$ to provide protection against chronic diseases [15]. The hypothesis of this study is that systematic doses of NOB or TAN (200 mg/kg/d) maintain the redox balance in the blood and liver, due to the increased availability of endogenous antioxidant compounds, which are spared with the supplementation of PMFs. For this purpose, blood concentrations of $\alpha$-tocopherol (vitamin E) and retinol (vitamin A derivative) were measured, as well as the levels of hepatic glutathione peroxidase (GPx), and the antioxidant effect of NOB and TAN on lipid peroxidation and total antioxidant capacity in the blood. The second objective was to quantify the hepatic production of NOB and TAN metabolites, which are eventually associated with health benefits.

\section{Materials and methods}

\subsection{Purification of Tangeritin and Nobiletin}

Cold pressed orange oil residues containing around 10\% PMFs, obtained from a local flavoring company, were initially purified by silica gel column chromatography (Biotage), using linear gradients of hexane and ethyl acetate for partial separation of tangeretin [(TAN) 5,6,7,8,4'-pentamethoxy-flavone] and nobiletin [(NOB) $5,6,7,8,3^{\prime}, 4^{\prime}$-hexamethoxyflavone]. Purification of TAN and NOB compounds were obtained by reverse phase liquid chromatography (RediSep C18 Reverse Phase) with linear gradients of methanol/aqueous formic acid, followed by crystallization with acetone and hexane. The purity of TAN and NOB was $>95 \%$ compared to authentic standards using methods previously reported [16]. The diagram in Figure 1 briefly describes the purification process.

\subsection{Animals and treatment}

Thirty male rats (Wistar) weighing 150-200 g from the Animal Center of São Paulo State University (UNESP) were housed in individual metabolic cages and were adapted for 7 days to the environmentally controlled temperature (23 $\pm 1{ }^{\circ} \mathrm{C}$ ), humidity $(55 \pm 5 \%)$ and a $12 \mathrm{~h}$ light/dark cycle. Rats had free access to water and a commercial diet (Presence Nutricao Animal ${ }^{\circledR}$ ) throughout the 15 days of the experiment under the same conditions. The basic composition of the rat chow contained: bran of soybean, wheat, and rice; ground whole corn; refined soybean oil; meat and fish meal; dextrin; sodium chloride; calcium iodate; and trace minerals and vitamins. High-Performance Liquid Chromatography (HPLC) analysis of the commercial rat chow showed retinol and $\alpha$-tocopherol occurred at concentrations of 0.04 and 1.10 mg per $20 \mathrm{~g}$ of commercial chow, respectively. This protocol was conducted in compliance with recommendations of the Brazilian College of Animal Experimentation (COBEA) and the experimental procedures received prior institutional approval by the Ethical Board for Animal Experimentation, School of Pharmaceutical Sciences, UNESP (Protocol \# 68/2015). 


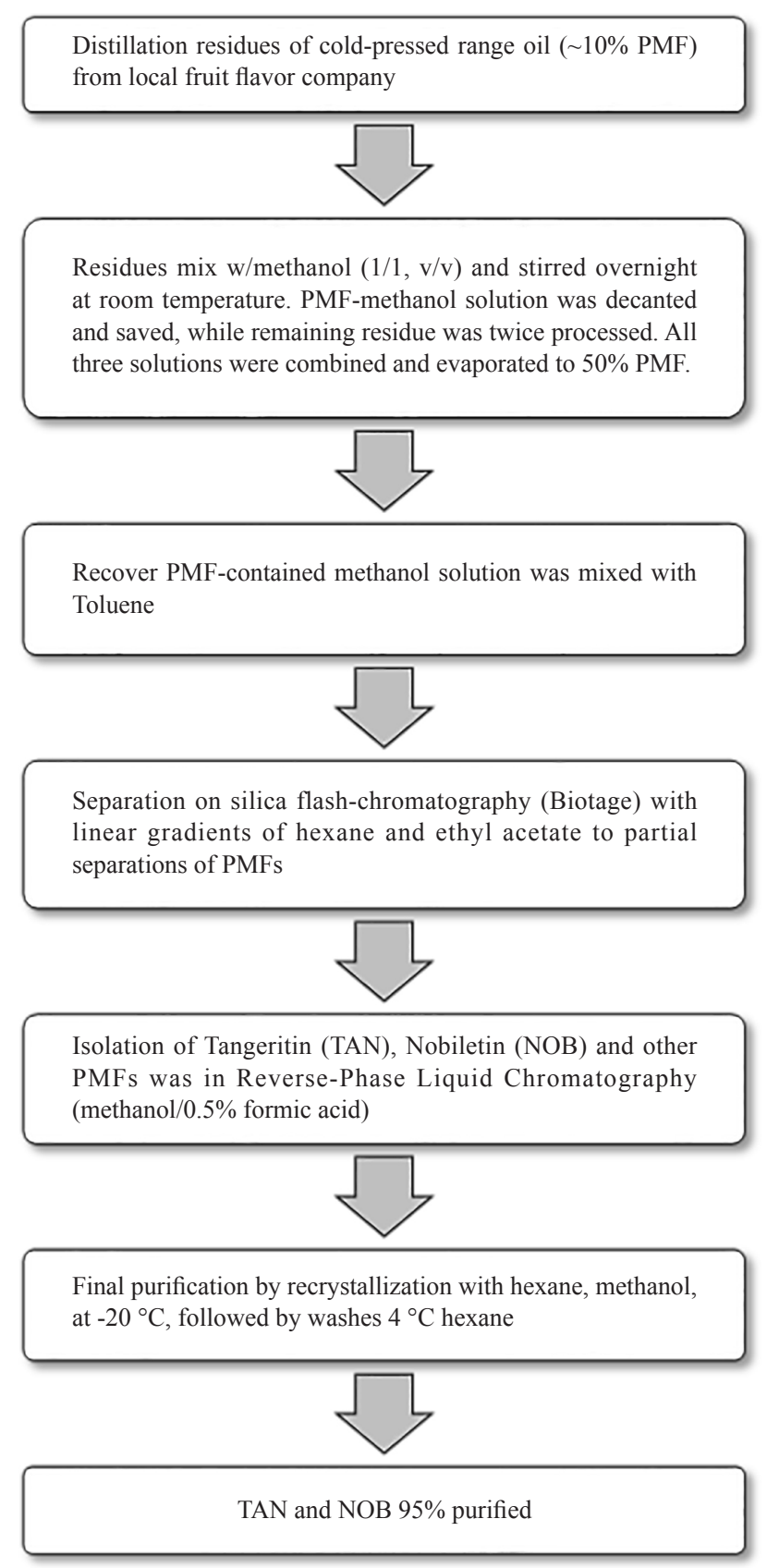

Figure 1. Flowchart of Nobiletin (NOB) and Tangeretin (TAN) purification by reverse phase liquid chromatography (adapted from Gonçalves et al, 2019)

\subsection{Experimental design}

The purified TAN and NOB were mixed separately with commercial plain yogurt $\left(\mathrm{Nestl}^{\mathbb{B}}\right)$ with a homogenizer operating at $820 \times \mathrm{g}$ for 10 minutes at a controlled ambient temperature $\left(25^{\circ} \mathrm{C}\right)$ (Figure 2). After the adaptation period, rats were assigned into three groups according to similar mean values of body weight: control group $(\mathrm{n}=10)$, NOB group $(\mathrm{n}=10)$, and TAN group $(\mathrm{n}=10)$. Each experimental group was treated with $200 \mathrm{mg} / \mathrm{kg} /$ day of NOB or TAN mixed in $1 \mathrm{~mL}$ plain yogurt by gavage once a day at 4:30 PM. The selected dose of $200 \mathrm{mg} / \mathrm{kg}$ bw/day in the current study was based on the protective effects of NOB and TAN against induced brain and liver damage in rats without 
toxic events $[6-7,9]$. The control group received $1 \mathrm{~mL}$ of plain yogurt (Nestlé) as a placebo. Body weight was checked every three days for the PMF dose adjustment. After 15 days of treatment, the rats were euthanized by decapitation and blood samples were collected in heparinized tubes (Hemofol ${ }^{\circledR}, 5000 \mathrm{UI} / \mathrm{mL}$, Brazil). Blood serum was obtained by centrifugation $\left(10,000 \times \mathrm{g}\right.$ for $15 \mathrm{~min}$ at $\left.25^{\circ} \mathrm{C}\right)$, and livers were removed and frozen $\left(-80^{\circ} \mathrm{C}\right)$ until further analysis.

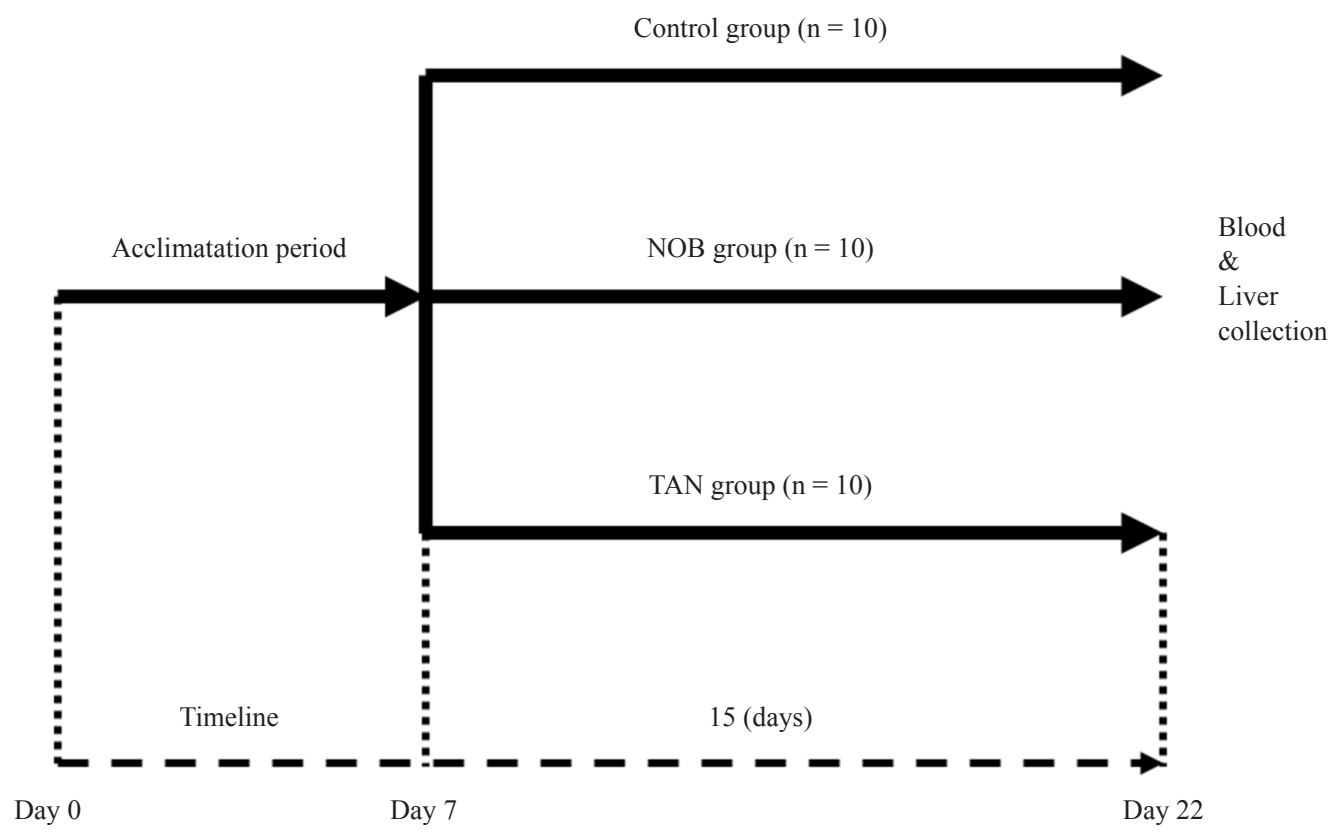

Figure 2. Experimental design. After the acclimatation period (7 days), Nobiletin (NOB) and Tangeretin (TAN) groups received, by gavage once a day a solution of NOB or TAN $(200 \mathrm{mg} / \mathrm{kg}$ ) in $1 \mathrm{ml}$ of vehicle (plain yogurt). Control group received $1 \mathrm{~mL}$ of vehicle for 15 days.

\subsection{Antioxidant capacity in the blood serum and liver}

Blood serum oxidative stress was measured by comparing levels of serum lipid peroxidation using thiobarbituric acid-reactive substances (TBARS) and quantified as $\mu \mathrm{M}$ malondialdehyde (MDA) [17]. Malondialdehyde (MDA) is the end product of lipid peroxidation and reacts with thiobarbituric acid (TBA) to form the MDA-TBA adduct in a 1:2 ratio with TBA. The absorbance of each test was obtained in a 96-well microplate reader at $540 \mathrm{~nm}$. Total antioxidant capacity in blood serum was measured as the Trolox equivalent antioxidant capacity (TEAC) using the 2,2'-azinobis (3-ethylbenzothiazoline-6-sulfonic acid (ABTS) assay [18]. The absorbance was measured at 734 nm to verify the formation of $\mathrm{ABTS}^{+}$and, to prepare the calibration curve, Trolox (Sigma) was used as a standard. All analyzes were performed in triplicate.

To assess liver oxidative stress, the organs were thawed and one gram of each was homogenized in $4 \mathrm{~mL}$ of $1.15 \%$ potassium chloride at $4{ }^{\circ} \mathrm{C}$, centrifuged $\left(10,000 \times \mathrm{g}\right.$ for $10 \mathrm{~min}$ at $\left.4{ }^{\circ} \mathrm{C}\right)$, and the supernatants collected. Levels of proteins in liver supernatants were determined using bovine serum albumin as standard for the assay [19]. Liver MDA values were given in $\mu \mathrm{M}$ MDA per mg protein. Liver glutathione peroxidase (GPx) activity was determined and the results were expressed as mmol of NADPH consumed $/ \mathrm{min} / \mathrm{mg}$ protein (liver) [20]. Bovine serum albumin was also used as the protein standard in the glutathione peroxidase (GPx) activity.

\subsection{Analysis of retinol, $\alpha$-tocopherol, and $\beta$-carotene in the blood serum}

Retinol ( $\geq 95 \%$ pure), $\alpha$-tocopherol ( $\geq 95 \%$ pure), and $\beta$-carotene $\left(\geq 95 \%\right.$ pure) were purchased from Sigma ${ }^{\circledR}$. This analysis and preparation of the standards and blood samples were according to [21]. Sample preparation was in glass tubes, cover with aluminum foil to minimize light-induced degradation of vitamins. Blood serum samples $(200 \mu \mathrm{L})$ were 
combined with methanol $(600 \mu \mathrm{L})$, thoroughly mixed, and centrifuged at $10,000 \times \mathrm{g}$ for 4 min at room temperature. Supernatants were collected and reduced to dryness under nitrogen flow, and the residues were dissolved into the mobile phase. Chromatographic analysis was performed with Ultra-Performance Liquid Chromatograph (UPLC) (Shimadzu Nexera X2), equipped with a photodiode array UV-Vis detector (PDA-SPD-M20A), auto sampler (Shimadzu Nexera X2 SIL-30 AC), and column compartment/heater, both enabling the control of the temperature. An Acquity UPLC $\mathrm{C}_{18}$ column $(2.0 \mathrm{~mm} \times 200 \mathrm{~mm}, 2.2 \mu \mathrm{m}$ particles $)$ was used for separation. Isocratic acetonitrile-methanol-dichloromethane $(75: 5: 20, \mathrm{v} / \mathrm{v} / \mathrm{v})$ was used as mobile phase; the flow rate was $0.3 \mathrm{~mL} / \mathrm{min}$. UV detection was accomplished at $325 \mathrm{~nm}$ for retinol, $450 \mathrm{~nm}$ for $\beta$-carotene, and $292 \mathrm{~nm}$ for $\alpha$-tocopherol [21]. Injection volume was $7 \mu \mathrm{L}$ and the compounds were separated within $10 \mathrm{~min}$. Data were collected and processed by LabSolutions Shimadzu Corporation software.

\subsection{Detection of liver PMF metabolites}

To analyze the metabolites in rat liver, $2.0 \mathrm{~mL}$ of methanol was added to each tube containing the complete freezedried supernatant of $1.0 \mathrm{~g}$ of homogenized tissue. The methanol-added samples were dispersed in a vortex at high speed for $2 \mathrm{~min}$. The samples were centrifuged for $10 \mathrm{~min} \mathrm{10,000 \times g}$ at room temperature under vacuum in a Savant concentrator. The clear supernatants were placed into individual $4 \mathrm{~mL}$ vials. The remaining sample precipitates were reextracted using the above procedure. The first and second supernatants were combined and evaporated at $60{ }^{\circ} \mathrm{C}$ under vacuum to near dryness then mixed with methanol to $2.0 \mathrm{~mL}$ and clarified through a 0.45 -micron PTFE filter. Detection and analysis of the metabolites in the liver samples were performed by HPLC (Waters 2695 Alliance ${ }^{\circledR}$ ), connected in parallel with a Photodiode Array Detector (PDA), and a Micromass ZQ quadrupole Mass Spectrometer with an electrospray ionization source (ESI), using a Waters $\mathrm{C}_{8}$ XBridge $(4.6 \times 150 \mathrm{~mm})$ column, using linear gradients of aqueous $0.5 \%$ formic acid and acetonitrile, (90/10; v/v), as described previously [22]. Detection of the NOB and TAN metabolites were by positive ion ESI-MS using single ion monitoring and by UV absorbance with a Waters 996 diodearray detector. Rat liver metabolites were quantified by comparisons with previously purified 4'-hydroxy-NOB, NOB4'-glucuronide, 4'-desmethyl TAN and Tan-4'-O-glucuronide standards [23].

\subsection{Statistical analysis}

The results of metabolites in rat livers are described in table and chromatogram charts. The variables to assess lipid peroxidation and antioxidant capacity in rat blood serum and liver were expressed as mean $\pm \mathrm{SD}$, and differences were considered statistically significant when $\mathrm{p} \leq 0.05$. Data were statistically compared using one-way analysis of variance (ANOVA) and SIDAK as post-hoc. Analyses were performed in SPSS Statistics software (v.21, SPSS: An IBM Company, Chicago, IL).

\section{Results}

\subsection{Body weight and food intake}

At the beginning of the supplementation period, there were no differences among body weights of the groups: control, TAN, and NOB (Figure 3), but on the 7th day, the rats supplemented with TAN had a higher body weight than the controls, while the weight of NOB rats was intermediate between the TAN and controls. At the end of the experiment (15th day), the rats treated with TAN gained 26\% more weight in comparison to NOB and controls (Figure 3 and Table 1). At the end of the experiment, the animals with the highest body weight were also those that ingested the highest daily and total amount of food (Table 1 and Figure 3). Consequently, they also had higher energy consumption (Table 1), as all rats were fed ad libitum with the same diet. Therefore, the discrepancy in weight gain between TAN and the other groups may be based on the greater appetite of these individuals, or on other unmeasured variables. Although the sample size was calculated taking into account differences within and between groups (ANOVA one-way), there are limitations in interpreting unexpected results, as the estimate is based on the main hypothesis previously determined. 


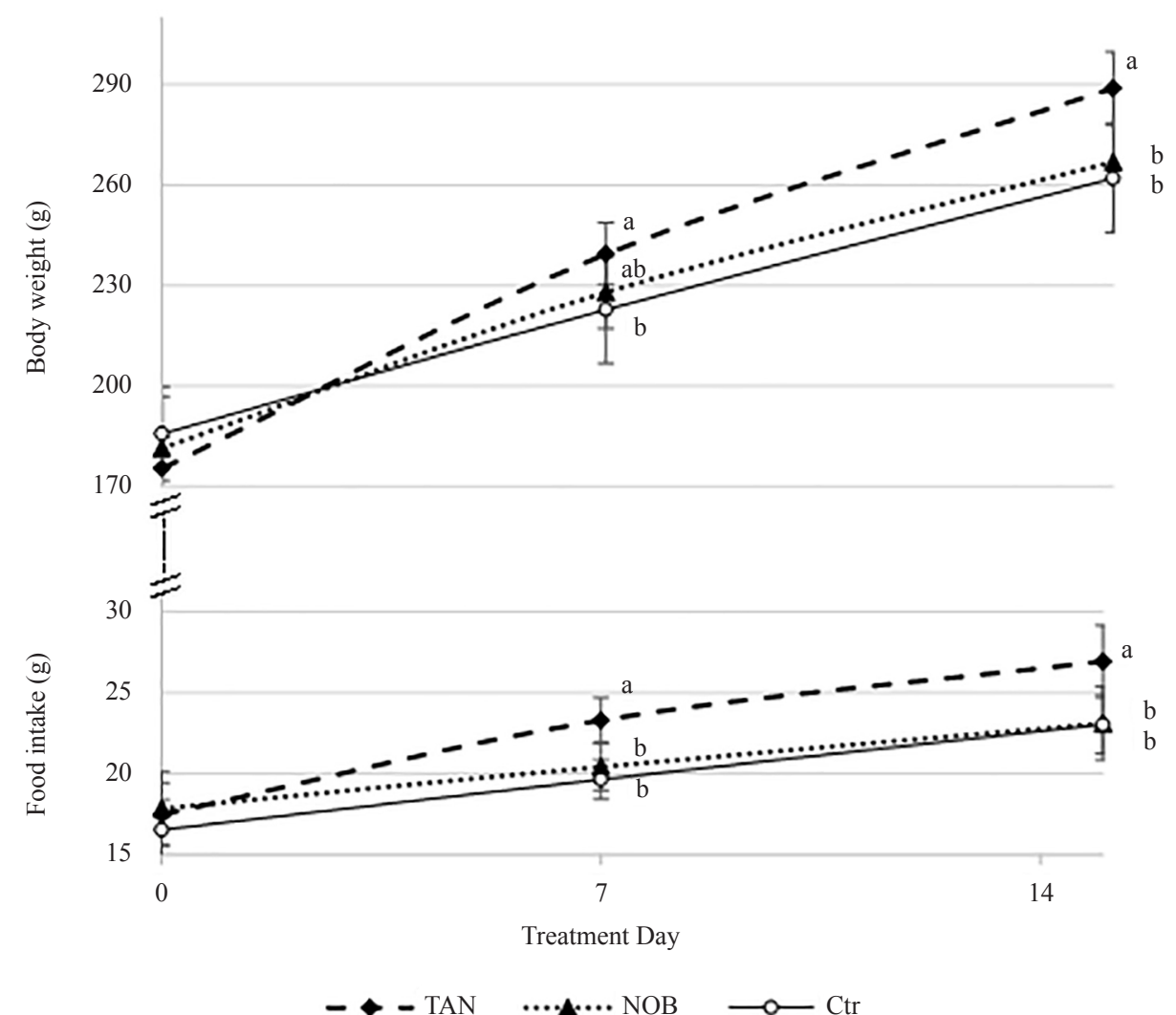

Figure 3. Body weight and food intake of rats treated with standard diet (control group), and diet supplemented with $200 \mathrm{mg} / \mathrm{kg}$.bw of tangeritin (TAN group) or nobiletin (NOB group) during the experimental period of 15 days

Table 1. Effect of NOB and TAN on body weight, food and energy intake in rats fed with normal caloric diet (AIN-93)

\begin{tabular}{cccc}
\hline & Control & NOB & TAN \\
\cline { 2 - 4 } Group & $(\mathrm{n}=10)$ & $(\mathrm{n}=10)$ & $(\mathrm{n}=10)$ \\
\hline Initial Weight (g) & $186 \pm 14^{\mathrm{a}}$ & $181 \pm 15^{\mathrm{a}}$ & $175 \pm 9^{\mathrm{a}}$ \\
Final Weight (g) & $262 \pm 16^{\mathrm{b}}$ & $267 \pm 21^{\mathrm{b}}$ & $289 \pm 11^{\mathrm{a}}$ \\
Weight Gain (15 d) & $76 \pm 13^{\mathrm{a}}$ & $86 \pm 23^{\mathrm{a}}$ & $114 \pm 6^{\mathrm{b}}$ \\
Intake (g/d) & $21.4 \pm 2.4^{\mathrm{b}}$ & $21.8 \pm 1.9^{\mathrm{b}}$ & $25.1 \pm 2.6^{\mathrm{a}}$ \\
Energy Intake (kcal/d) & $81 \pm 9^{\mathrm{b}}$ & $83 \pm 7^{\mathrm{b}}$ & $96 \pm 10^{\mathrm{a}}$ \\
\hline
\end{tabular}

Data are presented as mean $\pm \mathrm{SD}$.

One-way analysis of variance (ANOVA) and SIDAK post-hoc. Different letters show that there is statistical difference between groups $(\mathrm{p} \leq 0.05)$

\subsection{Redox status and antioxidant capacity}

Both TAN and NOB administered to healthy rats reduced the blood serum MDA in comparison to the control group. MDA values decreased by an average of $22 \%$ with TAN, while NOB reduced the blood serum MDA by $18 \%$ $(\mathrm{p} \leq 0.05)$ (Table 2). The serum antioxidant activity tested by the ABTS + assay increased $3 \%$ in the NOB group $(\mathrm{p} \leq$ 0.05 ), while there was no change in the TAN group. Yet, $\alpha$-tocopherol increased $20 \%$, and retinol increased $59 \%$ in the group treated with TAN $(\mathrm{p} \leq 0.05)$, but NOB and control showed no changes (Table 2). Chromatographic analysis of 
blood serum did not detect $\beta$-carotene in samples from any group. Analysis of liver peroxidation showed no significant differences among the three groups. Interventions with either PMF had no effects on the GPx levels in the liver.

Table 2. Antioxidant capacity in the blood serum and liver of rats supplemented with daily dose ( $200 \mathrm{mg} / \mathrm{kg}$ bw) of tangeretin (TAN), nobiletin (NOB) or vehicle (Control)

\begin{tabular}{|c|c|c|c|c|}
\hline \multirow{2}{*}{ Tissue } & \multirow{2}{*}{ Antioxidants Parameters } & Control & NOB & TAN \\
\hline & & $(\mathrm{n}=10)$ & $(\mathrm{n}=10)$ & $(\mathrm{n}=10)$ \\
\hline \multirow{8}{*}{ Blood Serum } & $\mathrm{MDA}(\mu \mathrm{M} / \mathrm{L})$ & $1.48 \pm 0.40^{\mathrm{a}}$ & $1.21 \pm 0.26^{\mathrm{b}}$ & $1.15 \pm 0.39^{\mathrm{b}}$ \\
\hline & & $(\Delta \%)$ & $(-18 \%)$ & $(-22 \%)$ \\
\hline & $\operatorname{ABTS}(\mathrm{mM} / \mathrm{L})$ & $1.68 \pm 0.06^{\mathrm{b}}$ & $1.73 \pm 0.05^{\mathrm{a}}$ & $1.70 \pm 0.02^{\mathrm{ab}}$ \\
\hline & & $(\Delta \%)$ & $(3.0 \%)$ & $(1.2 \%)$ \\
\hline & $\alpha$-tocopherol $(\mu \mathrm{M})$ & $6.63 \pm 0.89^{b}$ & $6.18 \pm 1.72^{\mathrm{b}}$ & $7.97 \pm 1.04^{\mathrm{a}}$ \\
\hline & & $(\Delta \%)$ & $(-6.8 \%)$ & $(20 \%)$ \\
\hline & retinol $(\mu \mathrm{M})$ & $1.35 \pm 0.13^{\mathrm{b}}$ & $1.27 \pm 0.49^{b}$ & $2.14 \pm 0.47^{\mathrm{a}}$ \\
\hline & & $(\Delta \%)$ & $(-5.9 \%)$ & $(59 \%)$ \\
\hline \multirow{4}{*}{ Liver } & $\operatorname{MDA}(\mu \mathrm{M} / \mathrm{g})$ & $0.40 \pm 0.18^{\mathrm{a}}$ & $0.48 \pm 0.14^{\mathrm{a}}$ & $0.54 \pm 0.11^{\mathrm{a}}$ \\
\hline & & $(\Delta \%)$ & $(20 \%)$ & $(35 \%)$ \\
\hline & GPx (mmol/min/mg) & $0.08 \pm 0.02^{\mathrm{a}}$ & $0.06 \pm 0.01^{\mathrm{a}}$ & $0.07 \pm 0.03^{\mathrm{a}}$ \\
\hline & & $(\Delta \%)$ & $(-25 \%)$ & $(-13 \%)$ \\
\hline
\end{tabular}

Data are presented as mean $\pm \mathrm{SD}$.

Antioxidant variables: MDA (malondialdehyde), ABTS (2,2'-azino-bis(3-ethylbenzothiazoline-6-sulfonic acid, $\alpha$-tocopherol), $\alpha$-tocopherol (vitamin E) and retinol (derivative of vitamin A) were measured in the blood serum, while the activity of MDA and GPx (glutathione peroxidase) were measure in the liver.

$\Delta \%=$ difference $\%$ between experimental group (NOB or TAN) versus Control.

One-way analysis of variance (ANOVA) and SIDAK post-hoc. Different letters show that there is statistical difference between groups ( $\mathrm{p} \leq 0.05$ ).

\subsection{PMF metabolites in rat liver}

Profiles of glucuronide and sulfate conjugates, as well as hydroxylated aglycone metabolites in the livers of TANand NOB-fed rats were analyzed by HPLC-ESI-MS and PDA (UV) detection [22]. Protonated molecular masses ([M $+\mathrm{H}^{+}$) and retention times of these compounds are shown in Table 3, and the MS and UV chromatograms are shown in Figure 4. The ESI-MS of the conjugated compounds exhibited neutral losses of 176 atomic mass units (amu), suggesting losses of glucuronic acid substituents, or $80 \mathrm{amu}$ for the loss of a sulfate group.

HPLC-MS analysis of the individual liver extracts showed two TAN metabolites: T1 (TAN-4'-O-glucuronide) at an average liver concentration of $0.011 \pm 0.0046 \mu \mathrm{g} / \mathrm{g} \mathrm{FW}$ and T2 (4'-desmethyl TAN, i.e., 4'-hydroxy-5,6,7,8tetramethoxyflavone) at an average liver concentration of $0.031 \pm 0.0054 \mu \mathrm{g} / \mathrm{g}$ FW. Trace levels of other metabolites exhibiting $359 \mathrm{~m} / \mathrm{z}$ fragment ions were also detected in a pooled and concentrated liver extract (data not shown), but were not quantifiable in the individual liver extracts. HPLC-MS analyses of the individual liver extracts additionally showed eight NOB metabolites, N1-N8 (Table 3), and most of them were similar to those previously reported in rat blood serum [24]. The ESI-MS, elution time, and concentration of each of these compounds, as well as their suggested molecular compositions, are listed in Table 3.

From these measured concentrations, total amounts of the cumulative TAN metabolites $(0.042 \mu \mathrm{g} / \mathrm{g} \mathrm{FW})$, occurred at 170 times lower than the cumulative levels of the NOB metabolites ( $7.18 \mu \mathrm{g} / \mathrm{g} \mathrm{FW})$. A substantial portion of the NOB metabolites occurred as a NOB-O-sulfate (N5) at $2.74 \mu \mathrm{g} / \mathrm{g} \mathrm{FW}$. Also, unlike TAN, NOB metabolism in the rat livers produced measurable levels of di-desmethyl metabolites $\left[(\mathrm{M}+\mathrm{H})^{+}(389)-2(\mathrm{CH} 2)\right]=375 \mathrm{~m} / \mathrm{z}$, including metabolites N4A and N4B. Of the NOB metabolites, most occurred as conjugates of glucuronic acid and sulfate, and a smaller portion 
of the metabolites occurred as free aglycones (a cumulative average of $1.75 \mu \mathrm{g} / \mathrm{g} \mathrm{FW}$ ). Only trace levels of the original NOB and TAN were detected in the individual liver extracts.
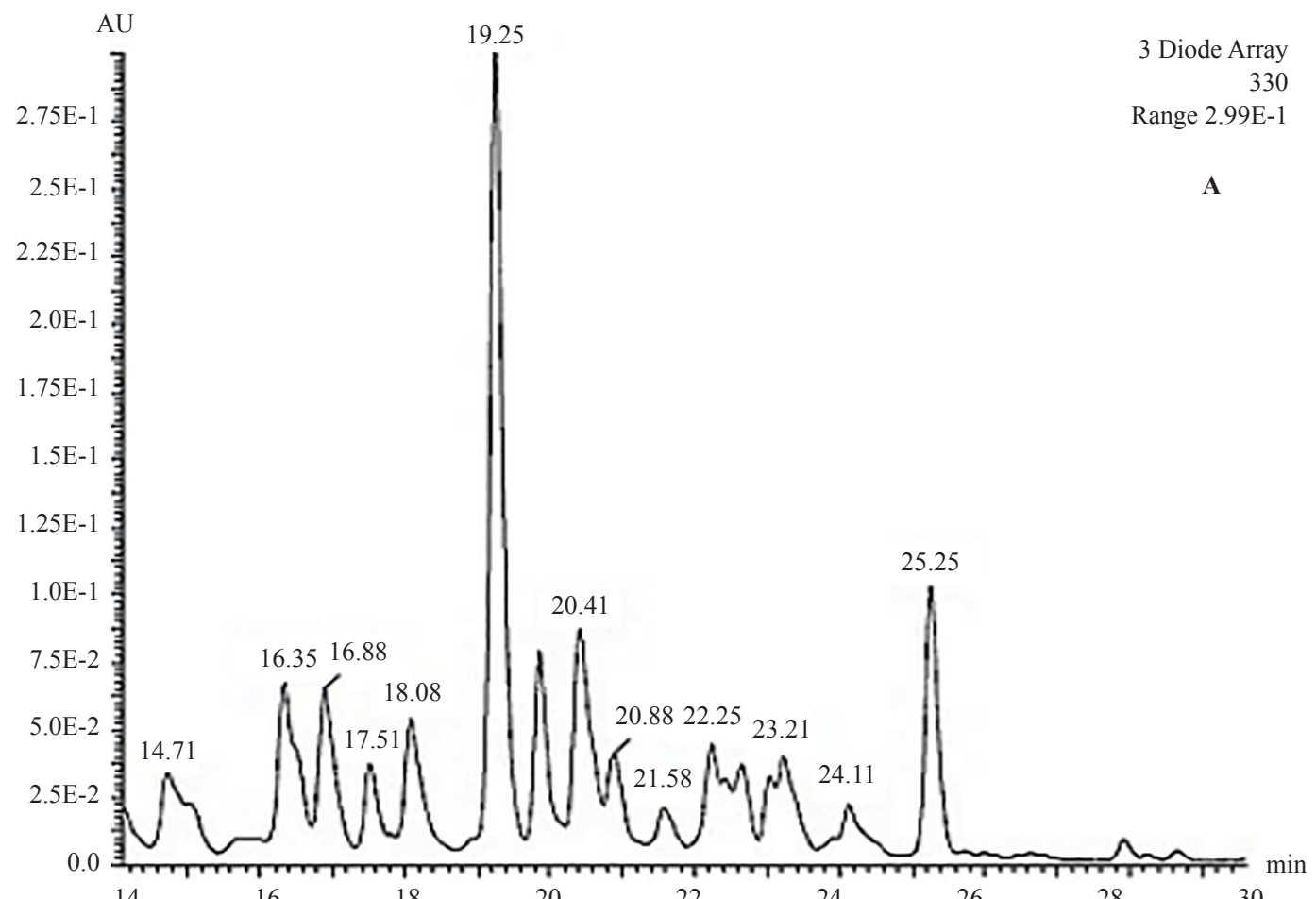

1416

18

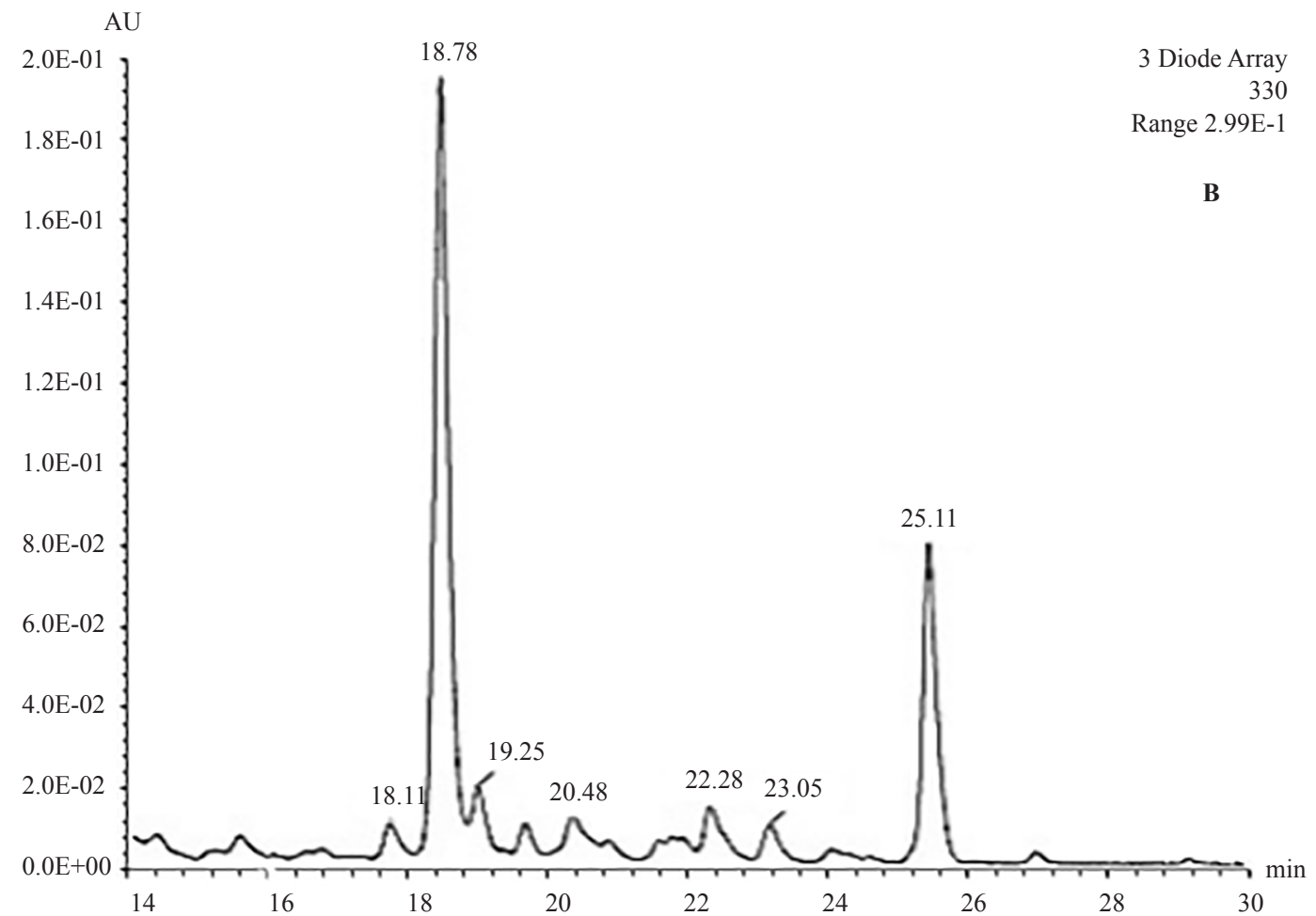

Figure 4. Profile of UV chromatogram (330 nm) of NOB (A) and TAN (B) metabolites in the rat's liver. The molecular masses of the ions (M + H) hydroxylated, glucuronide, and sulfated are listed in Table 2 
Table 3. Proposed structure, elution time and concentration of liver metabolites of rats supplemented with nobiletin (NOB) and tangeretin (TAN)

\begin{tabular}{ccccc}
\hline Metabolites & Proposed Structure & {$[\mathrm{M}+\mathrm{H}]^{+1}$ ESI-MS } & Elution time $(\mathrm{min})$ & Liver $(\mu \mathrm{g} / \mathrm{g})$ \\
\hline NOB 1 & NOB-O-glucuronide & $565 / 389$ & 20.3 & $1.18 \pm 0.64$ \\
NOB 2 & NOB-O-glucuronide & $565 / 389$ & 20.7 & $1.39 \pm 0.58$ \\
NOB 3 & NOB-O-glucuronide & $565 / 389$ & 17.6 & $0.12 \pm 0.09$ \\
NOB 4 & di-desmethyl NOB & 375 & 21.8 & $0.10 \pm 0.05$ \\
NOB 5 & NOB-O-sulfate & $469 / 389$ & 22.9 & $2.74 \pm 1.22$ \\
NOB 6 & mono-desmethyl NOB & 469 & 23.3 & $0.64 \pm 0.29$ \\
NOB 7 & mono-desmethyl NOB & 469 & 24.8 & $0.15 \pm 0.11$ \\
NOB 8 & mono-desmethyl NOB & 455 & 25.8 & $0.86 \pm 0.44$ \\
& & & 19.7 & $\Sigma_{\text {NOB }}=7.18$ \\
TAN 1 & TAN-4- $O$ '-glucuronide & 359 & 25.8 & $0.011 \pm 0.005$ \\
TAN 2 & 4'- $O$-hyroxy-5,6,7,8-pentame- & & & $0.031 \pm 0.005$ \\
& thoxy flavone & 535 & & $\Sigma_{\text {TAN }}=0.042$ \\
\hline
\end{tabular}

NOB $(1,2,3,4,5,6,7$ and 8$)$ : Eight metabolites detected in the liver of NOB-treated rats. Two metabolites are hydroxylated aglycones with protonated molecular masses of 389 ати (NOB 1) and 375 атu (NOB 4), two conjugated with glucuronic acid, 565 amu (NOB 3) and 551 amu (NOB 4), and four conjugated with sulfate groups, 469 ати (NOB 5, NOB 6, NOB 7) and 455 ати (NOB 8).

TAN (1 and 2): Two metabolites detected in the liver of animals supplemented with TAN identified as hydroxylated aglycone with protonated molecular masses of $359 \mathrm{amu}$ (TAN 1) and one glucuronic acid conjugate, $535 \mathrm{amu}$ (TAN 2).

\section{Discussion}

This study showed that systematic doses of NOB or TAN $(200 \mathrm{mg} / \mathrm{kg} / \mathrm{d})$ as dietary supplements for rats during a short time interval (15 days) reduced lipid peroxidation by $20 \%$ in circulating blood. In addition, a significant increase in the blood antioxidant capacity was observed after NOB treatment $(3 \%, \mathrm{p} \leq 0.05)$. At the end of the supplementation period, significant levels of NOB and TAN metabolites were detected in the liver, but not the parental compounds, suggesting that they were biotransformed before, or soon after, reaching the organ. The antioxidant activity measured in the hepatic tissue did not change with either supplement, but higher levels of retinol and $\alpha$-tocopherol were detected in the blood after TAN oral administration.

Animals treated with NOB had weight gain similar to the control group fed a standard diet, but the TAN group had higher intake and consequently higher body weight at the end of the experiment. Supplementation of NOB and TAN, or placebo, was performed by gavage and therefore had no direct effect on the flavor of the food. This is important because NOB and TAN are very bitter compounds [25]. Feng et al. [26] suggested an anti-obesity effect of TAN due to lower weight gain and body fat in rats that consumed $0.04 \%$ and $0.08 \%$ TAN mixed with a high-fat diet. Compared to the current experiment, these doses were 4.6 to 9.1 times higher, the initial body weight was $50 \%$ higher and, the intervention was longer ( $6 \mathrm{wk}$ ). In contrast, Nery et al. [27] found similar results to the current study in mice fed a highfat diet plus $100 \mathrm{mg} / \mathrm{kg}$ bw TAN for 4 weeks. The authors showed an increase in weight gain compared to the standard diet, but no change in animals fed a high-fat diet without TAN.

It has been recognized that the biological performance of flavonoids is dependent on their bioavailability, which can be very low due to the hydrophobic nature of these compounds. Glycosylated flavonoids, such as hesperidin, naringin, and eriocitrin, are mainly absorbed in the distal portion of the large intestine (colon), after deglycosylation by bacteria from the intestinal microbiota [23]. In contrast, polymethoxylated aglycones, such as NOB and TAN, show higher oral bioavailability than flavanones due to the lipophilic nature of multiple methoxy groups that decrease their hydrophilicity [28]. Such compounds are absorbed in the small intestine by passive diffusion, undergoing oxidative demethylation by cytochrome P450 in the intestinal wall (phase I metabolism), and can be conjugated by UGTs and SULTs enzymes 
(phase II metabolism). In the liver, phase II enzymes can produce glucuronate, sulfate, or methylate metabolites, which are much more hydrophilic. Part of them is secreted into the bile, performing the enterohepatic cycle, which increases the residence time and the effect of these compounds. Hepatic metabolites of NOB and TAN reach the bloodstream and peripheral tissues, and finally, they are eliminated by the urinary system or feces [2].

NOB and TAN are the main fraction of PMFs in the citrus peel, representing about $95 \%$ of the total PMF in balanced quantities [29]. However, they differ in bioaccessibility and absorption, with NOB being more bioavailable than TAN [5, 30]. Previously, we have shown that oral administration of $50 \mathrm{mg} / \mathrm{kg}$ bw of NOB, solubilized in corn oil, produced a higher content of metabolites in the blood of rats than the same dose of TAN, suggesting greater bioavailability of NOB [31]. We also showed that NOB produced eight metabolites, four glucuronides, and four aglycones, but TAN produced only two, an aglycone and a glucuronic acid conjugate [31].

In the present study, we detected TAN and NOB metabolites in the livers of each supplemented group, demonstrating that oral administration of $200 \mathrm{mg} / \mathrm{kg}$ bw of TAN or NOB was bioaccessible. Chromatographic analysis of the liver, obtained from all groups, revealed two metabolites of TAN, a hydroxylated aglycone, and a glucuronide metabolite, while eight metabolites of NOB were detected, four hydroxylated aglycones, three glucuronides and one sulfate metabolite, but no parental compounds. Other authors have described similar quantities of TAN and NOB metabolites [5, 31-33]. In our study, a 170 times higher concentration of total NOB metabolites in the liver was found in comparison to TAN metabolites. These results suggest that NOB metabolites presumably reached the bloodstream improving the antioxidant capacity in the blood, while very low levels of TAN metabolites were transported out of liver cells.

Some recent work has focused on the activity of the main metabolites of NOB and TAN, such as 5-dimethyl NOB [5-hydroxy-6,7,8,3',4'-pentamethoxyflavone) and 5-dimethyl TAN (5-hydroxy-6,7,8,4'-tetramethoxyflavone). These compounds decreased lipid peroxidation and ROS production in Saccharomyces cerevisiae, a eukaryote model under oxidative stress [5]. Another study showed the anti-inflammatory effect of the most abundant colonic metabolite of NOB in mice, 3',4'-didemethyl NOB (3',4'-dihydroxy-5,6,7,8-tetramethoxyflavone), which was able to inhibit NO production and iNOS and COX-2 gene expression, by suppression of nuclear translocation of NF- $\kappa \mathrm{B}$ and AP-1 transcription factors in RAW 264.7 macrophages [32].

Assessing the activity of antioxidant compounds in control animals helps to understand how to avoid oxidative stress and consequent biochemical and physiological damage. This is one of the main benefits of dietary supplements that attract consumers who seek to prevent the degenerative diseases due to aging. In this sense, our results showed that healthy rats supplemented orally with NOB, increased the antioxidant capacity of the blood by $3 \%$, assessed by ABTS, while reducing lipid peroxidation by $18 \%$, assessed by blood MDA levels. In turn, the TAN did not show a significant change in the antioxidant capacity in the blood, but its intake was associated with a $22 \%$ reduction in serum MDA levels, suggesting a protective effect against lipid peroxidation. It is interesting to note that previously we had found similar protective effects against oxidative stress and inflammation from citrus flavanones to C57BL/6J mice fed a highfat diet [34].

The efficiency of oral intake of PMFs, with regard to the mechanism of action and safety, is another relevant topic to consider for their preventive use. Previous results indicate the benefits of NOB and TAN at a dose similar to this study, $200 \mathrm{mg} / \mathrm{kg}$ bw/day, without mentioning any toxic effects [6-7, 9]. However, these studies used experimental models of oxidative stress induced by chemical agents or diseases. For example, the neuroprotective role of TAN was investigated in an experimental model of pilocarpine-induced epilepsy in rats previously supplemented with 50, 100, or $200 \mathrm{mg} / \mathrm{kg}$ bw/day. It was observed that the TAN inhibited the lesions in a dose-dependent manner, and $200 \mathrm{mg} / \mathrm{kg}$ being the most effective dose to reduce neuronal loss, with the number of viable cells in the injured animals similar to the control group [9]. In another study, doses of 100 or $200 \mathrm{mg} / \mathrm{kg}$ bw/day of NOB administered intragastrically to rats for 9 days, before the induced ischemic brain injury, inhibited overproduction of pro-inflammatory cytokines, such as TNF- $\alpha$, IL-1b, IL-6 and NO, suggesting anti-inflammatory and neuroprotective effects [6]. Intraperitoneal injection with $200 \mathrm{mg} / \mathrm{kg}$ bw of alcoholic extract containing NOB and TAN significantly attenuated brain damage in a rat ischemiareperfusion model [7].

It was previously mentioned that in the absence of oxidative damage induction, TAN did not influence any redox balance marker in the liver after four weeks of intervention [35]. These results are in accordance with the present study, which found no changes in the MDA or GPx in the liver of TAN-treated animals. In addition, experimental models of 
injury induction by drugs or pathologies showed that supplementation with TAN or NOB provided protection to the liver and extrahepatic tissues, assessed by tissue and inflammatory markers. For example, restoration of superoxide dismutase (SOD), GPx, and catalase levels have been observed in the liver and other organs, and a reduction in systemic lipid peroxidation, with decreased levels of MDA and NO in the bloodstream, in mice previously treated with high doses of ethanol [3]. Pre-treatment with NOB in acute liver damage induced by concanavalin A in mice reduced the levels of liver enzymes alanine aminotransferase and aspartate transaminase, decreased the intracellular generation of ROS, and suppressed the release of inflammatory cytokines TNF- $\alpha$ and interferon gamma (IFN- $\gamma$ ) [24]. In another study with cisplatin-induced acute liver damage in rodents, TAN decreased TNF- $\alpha$ and interleukin 10 (IL-10) inflammatory markers, lipid peroxidation (MDA), inflammatory systemic NO responses and increased glutathione(GSH), and GPx activities, improving liver function [36]. It has been suggested that the mechanisms underlying TAN protection against hepatocyte-induced oxidative damage occur by the positive regulation of molecular signaling pathways, such as heme oxygenase 1 (HO-1), neurofibromatosis type 2 (Nrf2), NAD(P)H quinone dehydrogenase 1 (NQO-1) and mitogenactivated protein kinase (MAPK) [37].

NOB and TAN express greater anti-inflammatory potential than glycoside flavonoids, such as hesperidin and narirutin, with NOB having the greatest anti-inflammatory effect among all, and the apparent anti-inflammatory capacity of TAN is, very likely, due to its cytotoxicity [4]. NOB metabolites show antioxidant activity by inhibiting the production of intracellular ROS [38] and blocking the expression of the nitric oxide synthases (iNOS) and cyclooxygenase 2 (COX-2) protein in murine macrophages [32-33, 38]. In an inflammatory skin model, 3',4'-dihydroxy-5,6,7,8-NOB inhibited MAPK and improved intracellular signaling pathway important in regulating the cell cycle (PI3K/Akt), leading to negative regulation of nuclear factor kappa-light-chain-enhancer of activated B cells $(\mathrm{NF}-\kappa \mathrm{B})$ and decreasing the accumulation of ROS [39]. TAN metabolites were able to enhance antioxidant capacity and cell survival, as well as inhibit inflammatory responses through downregulation of NF-kB, TNF- $\alpha$, INOS signaling pathways [39], and up-regulation of Nrf2 expression that mediates these protective impacts [38].

Numerous studies confirm that exogenous antioxidants from dietary fruits and vegetables work together with endogenous antioxidants against oxidative stress [40]. Thus, it is expected that the supplements of TAN and NOB jointly with endogenous vitamins, such as $\alpha$-tocopherol and retinol, will help to balance the antioxidant status in animals. However, in the present study, increased levels of retinol and $\alpha$-tocopherol under TAN treatment, suggested that TAN may interfere in the balance of these vitamins. Previously it was shown that female rats exposed to dimethylbenz(a)anthracene (DMBA) induced breast cancer, underwent a chemotherapeutic effect with TAN, protecting tissue damage, reducing lipid peroxidation, increasing antioxidant enzymes, and preserving antioxidant compounds, such as vitamin $\mathrm{C}$ and $\mathrm{E}$ [41]. On the other hand, the higher levels of antioxidant vitamins can be associated with an unbalance of the circulating antioxidants, which suggested some degree of toxicity for TAN. In a very interesting study, a U-shaped curve for the toxicity of TAN was detected, meaning that repetitive lower doses of TAN (50 to $100 \mathrm{mg} / \mathrm{kg}$ bw) can be as toxic to cells and tissues as acute and larger doses (1000 to $3000 \mathrm{mg} / \mathrm{kg} \mathrm{bw}$ ). According to these authors, supplementation of potential consumers with TAN will require much more extensive evaluations to establish its safety profile [13].

\section{Conclusions}

This study showed that systematic doses of NOB and TAN reduced lipid peroxidation, but only NOB increased the antioxidant capacity in the blood. Significant levels of NOB and TAN metabolites were found in the liver, suggesting that the parent compounds were absorbed and biotransformed after oral ingestion. High blood levels of retinol and $\alpha$-tocopherol after ingestion of TAN showed a metabolic pathway distinct for this PMF. Therefore, we conclude that under physiological conditions, regular doses of TAN and NOB can improve antioxidant activity, but prolonged use of TAN should be treated with caution, and broader studies of liver toxicity at different doses should be further explored, including clinical studies.

\section{Acknowledgments}

Author thanks Coordination for Higher Education Staff Development (CAPES) of Brazil for the scholarship 
granted to Marilia C M Rodrigues for her Master Degree in Flood Science and Nutrition; Ana Lucia M. Nasser for the technical support; and Dr Elizabeth Baldwin (ARS-USDA) for the technical review of this study, and Veronica Cook for English review. We also thank the USDA for the supply of PMF compounds, the UNESP School of Pharmacy for the financial support.

\section{References}

[1] Pisoschi AM, Pop A. The role of antioxidants in the chemistry of oxidative stress: A review. European Journal of Medicinal Chemistry. 2015; 97: 55-74. Available from: https://doi.org/10.1016/j.ejmech.2015.04.040.

[2] Karn A, Zhao CY, Yang F, Cui JF, Gao Z, Wang MQ, et al. In-vivo biotransformation of citrus functional components and their effects on health. Critical Reviews in Food Science and Nutrition. 2020; 61(5): 756-776. Available from: https://doi.org/10.1080/10408398.2020.1746234.

[3] Choi BK, Kim TW, Lee DR, Jung WH, Lim JH, Jung JY, et al. Polymethoxyflavonoid-rich citrus aurantium extract ameliorates ethanol-induced liver injury through modulation of AMPK and Nrf2-related signals in a binge drinking mouse model. Phytotherapy Research. 2015; 29: 1577-1584. Available from: https://doi.org/10.1002/ptr.5415.

[4] Chen XM, Tait AR, Kitts DD. Flavonoid composition of orange peel and its association with antioxidant and anti-inflammatory activities. Food Chemistry. 2017; 218: 15-21. Available from: https://doi.org/10.1016/ j.foodchem.2016.09.016.

[5] Wang M, Meng D, Zhang P, Wang X, Du G, Brennan C, et al. Antioxidant protection of nobiletin, 5-demethylnobiletin, Tangeretin, and 5-demethyltangeretin from citrus peel in saccharomyces cerevisiae. Journal of Agricultural and Food Chemistry. 2018; 66(12): 3155-3160. Available from: https://doi.org/10.1021/acs. jafc.8b00509.

[6] Zheng Y, Bu J, Yu L, Chen J, Liu H. Nobiletin improves propofol-induced neuroprotection via regulating Akt/

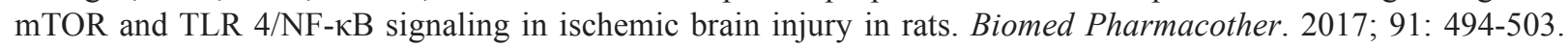
Available from: https://doi.org/10.1016/j.biopha.2017.04.048.

[7] Yang EJ, Lim SH, Song KS, Han HS, Lee J. Identification of active compounds from aurantii immatri pericarpium attenuating brain injury in a rat model of ischemia-reperfusion. Food Chemistry. 2013; 138(1): 663-670. Available from: https://doi.org/10.1016/j.foodchem.2012.09.137.

[8] Mulvihill EE, Burke AC, Huff MW. Citrus flavonoids as regulators of lipoprotein metabolism and atherosclerosis. Annual Review of Nutrition. 2016; 36: 275-299. Available from: https://doi.org/10.1146/annurevnutr-071715-050718.

[9] Guo XQ, Cao YL, Hao F, Yan ZR, Wang ML, Liu XW. Tangeretin alters neuronal apoptosis and ameliorates the severity of seizures in experimental epilepsy-induced rats by modulating apoptotic protein expressions, regulating matrix metalloproteinases, and activating the PI3K/Akt cell survival pathway. Advances in Medical Sciences. 2017; 62(2): 246-253. Available from: https://doi.org/10.1016/j.advms.2016.11.011.

[10] He Z, Li X, Chen H, He K, Liu Y, Gong J, et al. Nobiletin attenuates lipopolysaccharide/D galactosamine induced liver injury in mice by activating the Nrf2 antioxidant pathway and subsequently inhibiting $\mathrm{NF} \kappa \mathrm{B}$ mediated cytokine production. Molecular Medicine Reports. 2016; 14(6): 5595-5600. Available from: https://doi. org/10.3892/mmr.2016.5943.

[11] Evans M, Judy WV, Wilson D, Rumberger JA, Guthrie N. Randomized, double-blind, placebo-controlled, clinical study on the effect of Diabetinol(®) on glycemic control of subjects with impaired fasting glucose. Diabetes, Metabolic Syndrome and Obesity: Targets and Therapy. 2015; 8: 275-286. Available from: https://doi.org/10.2147/ DMSO.S79450.

[12] Salehi B, Martorell M, Arbiser JL, Sureda A, Martins N, Maurya PK, et al. Antioxidants: Positive or negative actors? Biomolecules. 2018; 8(4): 124. Available from: https://doi.org/10.3390/biom8040124.

[13] Ting Y, Chiou YS, Jiang Y, Pan MH, Lin Z, Huang Q. Safety evaluation of tangeretin and the effect of using emulsion-based delivery system: Oral acute and 28-day sub-acute toxicity study using mice. Food Research International. 2015; 74: 140-150. Available from: https://doi.org/10.1016/j.foodres.2015.04.031.

[14] Nair AB, Jacob S. A simple practice guide for dose conversion between animals and human. Journal of Basic and Clinical Pharmacy. 2016; 7(2): 27-31. Available from: https://doi.org/10.4103/0976-0105.177703.

[15] Ballard CR, Maróstica MR. Chapter 10-Health Benefits of Flavonoids. Bioactive Compounds-Health Benefits and Potential Applications. Woodhead Publishing; 2019. p.185-201. Available from: https://doi.org/10.1016/B978-012-814774-0.00010-4. 
[16] Gonçalves DR, Manthey JA, da Costa PI, Rodrigues MCM, Cesar TB. Analysis of fluorescence spectra of citrus polymethoxylated flavones and their incorporation into mammalian cells. Journal of Agricultural and Food Chemistry. 2018; 66(28): 7531-7541. Available from: https://doi.org/10.1021/acs.jafc.8b02052.

[17] Yagi K. Simple assay for the level of total lipid peroxides in serum or plasma. Methods in Molecular Biology. 1998; 108: 101-106.

[18] Janaszewska A, Bartosz G. Assay of total antioxidant capacity: comparison of four methods as applied to human blood plasma. Scandinavian Journal of Clinical and Laboratory Investigation. 2002; 62(3): 231-236. Available from: https://doi.org/10.1080/003655102317475498.

[19] Lowry OH, Rosebrough NJ, Farr AL, Randall RJ. Protein measurement with the Folin phenol reagent. Journal of Biological Chemistry. 1951; 193: 265-275.

[20] Rush JW, Sandiford SD. Plasma glutathione peroxidase in healthy young adults: influence of gender and physical activity. Clinical Biochemistry. 2003; 36(5): 345-351. Available from: https://doi.org/10.1016/s00099120(03)00039-0.

[21] Arnaud J, Fortis I, Blachier S, Kia D, Favier A. Simultaneous determination of retinol, alpha-tocopherol and betacarotene in serum by isocratic high-performance liquid chromatography. Journal of Chromatography A. 1991; 572(1-2): 103-116. Available from: https://doi.org/10.1016/0378-4347(91)80476-s.

[22] Pereira-Caro G, Ludwig IA, Polyviou T, Malkova D, García A, Moreno-Rojas JM, et al. Identification of Plasma and Urinary Metabolites and Catabolites Derived from Orange Juice (Poly)phenols: Analysis by High-Performance Liquid Chromatography High-Resolution Mass Spectrometry. Journal of Agricultural and Food Chemistry. 2016; 64: 5724-5735. Available from: https://doi.org/10.1021/acs.jafc.6b02088.

[23] Pereira-Caro G, Borges G, van der Hooft J, Clifford MN, Del Rio D, Lean ME, et al. Orange juice (poly)phenols are highly bioavailable in humans. The American Journal of Clinical Nutrition. 2014; 100(5): 1378-1384. Available from: https://doi.org/10.3945/ajcn.114.090282.

[24] Zhao J, Park S, Kim JW, Qi J, Zhou Z, Lim CW, et al. Nicotine attenuates concanavalin A-induced liver injury in mice by regulating the $\alpha 7$-nicotinic acetylcholine receptor in Kupffer cells. International Immunopharmacology. 2020; 78: 106071. Available from: https://doi.org/10.1016/j.intimp.2019.106071.

[25] Batenburg AM, de Joode T, Gouka RJ. Characterization and modulation of the bitterness of polymethoxyflavones using sensory and receptor-based methods. Journal of Agricultural and Food Chemistry. 2016; 64(12): $2619-2626$. Available from: https://doi.org/10.1021/acs.jafc.5b05833.

[26] Feng K, Lan Y, Zhu X, Li J, Chen T, Huang Q, et al. Hepatic lipidomics analysis reveals the antiobesity and cholesterol-lowering effects of tangeretin in high-fat diet-fed rats. Journal of Agricultural and Food Chemistry. 2020; 68(22): 6142-6153. Available from: https://doi.org/10.1021/acs.jafc.0c01778.

[27] Nery M, Ferreira PS, Gonçalves DR, Spolidorio LC, Manthey JA, Cesar TB. Physiological effects of tangeretin and heptamethoxyflavone on obese C57BL/6J mice fed a high-fat diet and analyses of the metabolites originating from these two polymethoxylated flavones. Food Science \& Nutrition. 2021; 9(4): 1997-2009. Available from: https://doi.org/10.1002/fsn3.2167.

[28] Hung WL, Chang WS, Lu WC, Wei GJ, Wang Y, Ho CT, et al. Pharmacokinetics, bioavailability, tissue distribution and excretion of tangeretin in rat. Journal of Food and Drug Analysis. 2018; 26(2): 849-857. Available from: https://doi.org/10.1016/j.jfda.2017.08.003.

[29] Wang R. Profile analysis and In vitro absorption study of citrus peel polymethoxyflavones. PhD Thesis. New Brunswick, New Jersey; 2015. Available from: https://rucore.libraries.rutgers.edu/rutgers-lib/48710/PDF/1/play/.

[30] Li S, Wang Z, Sang S, Huang MT, Ho CT. Identification of nobiletin metabolites in mouse urine. Molecular Nutrition \& Food Research. 2006; 50: 291-299. Available from: https://doi.org/10.1002/mnfr.200500214291.

[31] Manthey JA, Cesar TB, Jackson E, Mertens-Talcott S. Pharmacokinetic study of nobiletin and tangeretin in rat serum by high-performance liquid chromatography-electrospray ionization-mass spectrometry. Journal of Agricultural and Food Chemistry. 2011; 59(1): 145-151. Available from: https://doi.org/10.1021/jf1033224.

[32] Su JD, Yen JH, Li S, Weng CY, Lin MH, et al. 3',4'-didemethylnobiletin induces phase II detoxification gene expression and modulates PI3K/Akt signaling in PC12 cells. Free Radical Biology and Medicine. 2012; 52(1): 126-141. Available from: https://doi.org/10.1016/j.freeradbiomed.2011.10.002.

[33] Wu X, Song M, Rakariyatham K, Zheng J, Guo S, Tang Z, et al. Anti-inflammatory effects of 4'-demethylnobiletin, a major metabolite of nobiletin. Journal of Functional Foods. 2015; 19(Pt A): 278-287. Available from: https://doi. org/10.1016/j.jff.2015.09.035.

[34] Ferreira PS, Spolidorio LC, Manthey JA, Cesar TB. Citrus flavanones prevent systemic inflammation and ameliorate oxidative stress in C57BL/6J mice fed high-fat diet. Food \& Function. 2016; 7(6): 2675-2681. Available from: https://doi.org/10.1039/c5fo01541c. 
[35] Lakshmi A, Subramanian SP. Tangeretin ameliorates oxidative stress in the renal tissues of rats with experimental breast cancer induced by 7,12-dimethylbenz[a]anthracene. Toxicology Letters. 2014; 229(2): 333-348. Available from: https://doi.org/10.1016/j.toxlet.2014.06.845.

[36] Omar HA, Mohamed WR, Arab HH, Arafa E-SA. Tangeretin Alleviates Cisplatin-Induced Acute Hepatic Injury in Rats: Targeting MAPKs and Apoptosis. PLoS ONE. 2016; 11: e0151649. https://doi.org/10.1371/journal. pone.0151649.

[37] Liang F, Fang Y, Cao W, Zhang Z, Pan S, Xu X. Attenuation of tert-Butyl Hydroperoxide (t-BHP)-Induced Oxidative Damage in HepG2 Cells by Tangeretin: Relevance of the Nrf2-ARE and MAPK Signaling Pathways. Journal of Agricultural and Food Chemistry. 2018; 66(25): 6317-6325. Available from: https://doi.org/10.1021/ acs.jafc. $8 \mathrm{~b} 01875$.

[38] Li S, Sang S, Pan MH, Lai CS, Lo CY, Yang CS, et al. Anti-inflammatory property of the urinary metabolites of nobiletin in mouse. Bioorganic \& Medicinal Chemistry Letters. 2007; 17(18): 5177-5181. Available from: https:// doi.org/10.1016/j.bmcl.2007.06.096.

[39] Wu J, Zhao YM, Deng ZK. Tangeretin ameliorates renal failure via regulating oxidative stress, NF- $\kappa \mathrm{B}-\mathrm{TNF}-\alpha /$ iNOS signalling and improves memory and cognitive deficits in 5/6 nephrectomized rats. Inflammopharmacology. 2018; 26(1): 119-132. Available from: https://doi.org/10.1007/s10787-017-0394-4.

[40] Shakour ZTA, Fayek NM, Farag MA. How do biocatalysis and biotransformation affect Citrus dietary flavonoids chemistry and bioactivity? A review. Critical Reviews in Biotechnology. 2020; 40(5): 689-714. Available from: https://doi.org/10.1080/07388551.2020.1753648.

[41] Periyasamy K, Baskaran K, Ilakkia A, Vanitha K, Selvaraj S, Sakthisekaran D. Antitumor efficacy of tangeretin by targeting the oxidative stress mediated on 7,12-dimethylbenz(a) anthracene-induced proliferative breast cancer in Sprague-Dawley rats. Cancer Chemotherapy and Pharmacology. 2015; 75: 263. Available from: https://doi. org/10.1007/s00280-014-2629-z. 